\title{
Demonstration of a mental analog of an external rotation
}

\author{
LYNN A. COOPER \\ University of California, San Diego, La Jolla, California 92093
}

\begin{abstract}
Subjects imagined a designated two-dimensional shape rotating within a blank, circular field at a self-determined rate. At some point during the mental rotation, a test shape was presented at one of 12 picture-plane orientations, and the subject was required to determine as rapidly as possible whether the test shape was the same as the originally designated shape or was its mirror image. When the test shape was presented in the expected orientation (the orientation assumed to correspond to the current orientation of the rotating internal representation), reaction time was short and constant, regardless of the angular departure of that orientation from a previously trained position. This was true even when the test shape was presented in an orientation which had not previously been tested. When the test shape was presented at some other, unexpected orientation, reaction time increased linearly with the angular difference between the expected orientation and the orientation of the test shape. It is argued that these results provide a demonstration of the "analog" nature of mental rotation.
\end{abstract}

Recently, several investigators have reported that the time required to determine whether two visual stimuli are the same in shape or mirror images increases linearly with the angular difference between the orientations of the visual stimuli (Cooper, 1975; Cooper \& Shepard. 1973; Metzler, 1973; Metzler \& Shepard, 1974; Shepard \& Metzler, 1971). This linear relationship between reaction time and angular difference in orientation has led these investigators to suggest that subjects perform the task by "mentally rotating" an internal representation of one of the visual stimuli into congruence with the orientation of the other stimulus and then comparing the two representations for a match or a mismatch in shape.

Cooper and Shepard (1973). Metzler and Shepard (1974), and Shepard (1975) have viewed this process of mental rotation as an internal analog of the process that occurs when the rotation of an external object is perceived. Central to these investigators notions is the idea that, during a mental rotation, the internal process passes through a pathway or a trajectory. This trajectory can be viewed as a series of intermediate states, between the beginning and the end of the process, which have a one-to-one correspondence to the intermediate stages in the external rotation of an object. This one-to-one correspondence between the

This research was supported primarily by National Science Foundation Grant GB-31971X to Roger N. Shepard and also in part by National Institute of Mental Health Small Grant MH 25722-01 to the author. Roger N. Shepard provided extremely helpful discussion and advice during all stages of this research. Jim Cunningham and Jay McClelland provided valuable comments on the manuscript. Special thanks go to Peter Podgorny for his indispensable and enthusiastic assistance in running the subjects and analyzing the data and also for fruitful discussions concerning all aspects of this research. Requests for reprints should be addressed to: Lynn A. Cooper, Department of Psychology C-009. University of California. San Diego, La Jolla. California 92093. intermediate states in a mental rotation and a rotation of an external object need not be one of structural isomorphism between the internal representation underoing the mental rotation and the external object undergoing the physical rotation. Rather, the correspondence may entail only that. during a mental rotation. the internal process passes through a series of states at each one of which the subject is especially prepared for the presentation of a particular external object in a particular orientation.

The linear relationship between reaction time and angular difference in orientation. obtained in the studies mentioned above, is one form of evidence that the internal process underlying the observed reaction times is passing through an ordered series of stages. That is, the linear reaction-time function indicates that the time needed to compare two vistial objects presented at orientations $A$ and $C$ is an additive combination of the time needed to compare those objects presented at orientations $A$ and $B$ and the time needed to compare those objects presented at orientations $B$ and $C$. This finding is indirect evidence for the claim that the internal process underlying comparison of the objects presented in orientations $A$ and $C$ passes through an intermediate state corresponding to orientation $B$.

The objective of the present experiment is to provide additional. more conclusive evidence that the internal process underlying comparison of visual objects differing in orientation is specifically one of rotation, in that the successive states in the trajectory through which the process passes have a one-to-one correspondence to the successive stages in the rotation of an external object. This evidence consists of denonstrating that, while mentally rotating an internal representation of a visual shape from orientation $A$ to orientation $C$, the most rapid 
discriminative responses are obtained to an external test shape presented in an intermediate orientation $B$.

The present experiment incorporates and expands upon features of two previous studies designed to demonstrate this sort of correspondence between a mental rotation and an external rotation of a physical object lsee Cooper d Shepard. 1973. Experiment II. and Metaler. 1973). Specifically, subjects are instructed to imagine a designated visual shape rotating within a circular field at a self-determined rate (ct. Met/ler. 1973; Metzler \& Shepard. 1974). At some randomly determined point during the mental rotation. a test probe is presented and the subject is required to determine as rapidly as possible whether the test form is the same as the originally designated shape or its mirror image. On a portion of the trials. the test probe is presented in the "expected" orientation-viz. the external orientation assumed to be congruent with the orientation of the rotating internal representation of the designated shape (cf. Cooper \& Shepard, 1973. Experiment II). On the remainder of the trials, the test probe is presented in some other "unexpected" orientation. The points in time during the mental rotation at which test probes are presented are based on estimated rotation rates for the present subjects and present stimuli from a previous study (Cooper. 1975).

The significant novel feature of the present experiment is that on a small proportion of the trials. test probes are presented at intermediate orientations which are unfamiliar to the subjects. In the Cooper (1975) experiment, the present subjects viewed test forms at six equally spaced orientations about the circle. In the present experiment, test probes are introduced at orientations between these six familiar positions. If reaction times to "expected", probes presented in these intermediate orientations are as rapid as reaction times to "expected" probes presented at previously experienced orientations, then evidence for the analog nature of mental rotation. in the sense outlined above. is provided. Such an outcome would demonstrate that. in rotating from orientation $A$ to orientation $C$. the internal process does pass through an intermediate state corresponding to orientation $B$.

\section{METHOD}

\section{Subjects}

The six subjects were all students and staff at Stanford University. This group of subjects consisted of all of the original participants in a set of earlier. related experiments (Cooper. 1975) who were still residing in the Stanford area.

\section{Stimuli}

The stimuli were the eight random, angular shapes used by Cooper (1975). These two-dimensional shapes varied in the number of angles or points determining inflections on the perimeter, from a minimum of 6 points to a maximum of 24 points. For each shape. both an arbitrarily selected "standard" version and its mirror image or "reflected" version were included in the stimulus set.
Advance "identity" cues were also presented. Identity in formation consisted of an outline drawing of the standard version of the upcoming test form, displayed in a previously learned orientation. In the Cooper (1975) experiments, the present subjects had learned to discriminate standard versions of the shapes from reflected versions at one of six picture-plane orientations. This orientation constituted the previously learned or "trained" orientation in which the identity cue was presented. All stimuli were presented in an Iconix three-field tachistoscope and appeared centered within an illuminated circular field with a black surround. Luminance of the circular field was $68.5 \mathrm{~cd} / \mathrm{m}^{2}$, and it subtended a visual angle of $4^{\circ}$. Both the test forms and the identity cues subtended an angle of about $2^{\circ}$.

In order to reduce the number of trials per subject, eath subject was tested with only three of the eight possible shapes. Fotms were assigned randomly to individual subjects, with the constraint that the eight forms be represented approximately equally across subjects.

\section{Procedure}

Prior to the experimental trials, each subject was refamiliarized with both standard and reflected versions of the test shapes and completed 36 practice trials using the standard experimental procedure.

The sequence of events on each experimental trial was as follows: The subject was told that the trial was beginning, and the identity cue was displayed for $2 \mathrm{sec}$. The identity cue informed the subject as to the standard shape for that trial. and it was always presented in the trained orientation. Immediately following the offset of the identity cue. there was a 100 -msec blackout. This brief blackout informed the subject that he was to prepare to imagine the standard shape which had been presented as the identity cue rotating in a clockwise direction. A blank, circular field was then presented, and the subject began the mental rotation. At some preselected time after the initiation of the mental rotation, a test form was presented at one of 12 equally spaced orientations within the circular field. The subject determined as rapidly as possible whether the test shape was the same as the identity cue (a standard version) or its mirror image (a reflected version). "Standard" responses were signaled by pushing a right-hand button. and "reflected" responses were signaled by pushing a left-hand button. This response assignment was reversed for the one left-handed subject, so the preferred hand was always used to respond "standard."

There were tw'o types of experimental trials- "probe-expected" trials and "probe-unexpected" trials. On "probe-expected" trials. the test form was presented in the expected orientation, i.e., the orientation assumed to be congruent with the current orientation of the rotating internal representation of the identity cue. This was accomplished in the following manner. For each subject and each standard shape. rotation rates were computed from the data of Cooper's (1975) Experiment II. (These rates were determined by plotting mean reaction time as a function of angular departure of the test form from the trained orientation for each individual subject and each test shape. The slope of the best-fitting straight line was computed for each reaction-time function, and the reciprocal of the slope provided an estimate of rotation rate expressed as degrees per millisecond.) Each rotation rate was then used to determine the duration of the blank, circular "rotation" tield for a test form presented at a given orientation. Durations were thus computed for each individual subject, each standard shape and each test-form orientation. Consider, for example, a subject whose estimated rotation rate for a particular standard shape was $1.5^{\circ} / \mathrm{msec}$. For this subject, on a "probe-expected" trial on which the test form was presented at $120^{\circ}$ of clockwise rotation from the trained orientation, the duration of the blank, circular "rotation" field would have been $80 \mathrm{msec}$.

On the majority of the "probe-expected" trials ("probe-expected, familiar" trials). the test form was presented in one of six equally spaced orientations around the circle (in $60^{\circ}$ steps of clockwise rotation. starting from the trained orientation). The subjects were familiar with viewing the test forms in these positions. as only these six orientations were used in the Cooper (1975) experiments. On the remainder of the "probe-expected" trials ("probe-expected. 
untamiliar" trials), the test form was presented at an orientation in which the subjects had never before viewed the form. These unfamiliar orientations were the six intermediate $30^{\circ}$ steps between the familiar six $60^{\circ}$ steps of clockwise rotation. Thus, the unfamiliar orientations consisted of $30^{\circ}, 90^{\circ}, 150^{\circ}, 210^{\circ}, 270^{\circ}$, and $330^{\circ}$ angular departures from the trained orientation, and familiar orientations consisted of $0^{\circ}, 60^{\circ}, 120^{\circ}, 180^{\circ}, 240^{\circ}$. and $300^{\circ}$ angular departures from the trained orientation.

On "probe-unexpected" trials, the test form was presented in an orientation departing from the assumed current orientation of the rotating internal representation of the identity cue. Only the six familiar orientations were used on these "probe-unexpected" trials. The duration of the blank, circular "rotation" field and the angular departure of the test form from the expected orientation were computed from the rotation rates described above. Consider, again, the subject whose rotation rate for a particular shape was $1.5^{\circ}$ msec. For a "probe-unexpected" trial on which the test form was presented at $120^{\circ}$ of clockwise rotation from the trained orientation and at a $120^{\circ}$ counterclock wise angular departure from the expected orientation, the duration of the blank, circular "rotation" field would have been 160 msec.

Instructions to the subjects emphasized both speed and accuracy of the discriminative "standard-reflected" response. Subjects were instructed to begin the mental rotations as soon as the blank, circular "rotation" field appeared, and they were encouraged to perform the mental rotations at a natural, comfortable rate. Subjects were not told that test forms would sometimes appear in unfamiliar orientations.

\section{General Experimental Design}

Each of the six subjects completed 108 "probe-expected, familiar" trials, 36 "probe-expected, unfamiliar" trials, and 108 "probe-unexpected" trials. The "probe-expected. familiar" trials consisted of three presentations of each of the three standard shapes at each of the six familiar orientations in both standard and reflected versions. The "probe-expected, unfamiliar" trials consisted of one presentation of each combination of standard shape, unfamiliar orientation and test-form version. Thus, each of the "probe-expected, unfamiliar" trials was unique.

The "probe-unexpected" trials consisted of three presentations of each of the three standard shapes at each of the familiar orientations in both standard and reflected versions. The three presentations consisted of one presentation at each of three angular departures from the expected orientation. For any given expected orientation. the three angular departures were $60^{\circ}, 120^{\circ}$, and $180^{\circ}$.

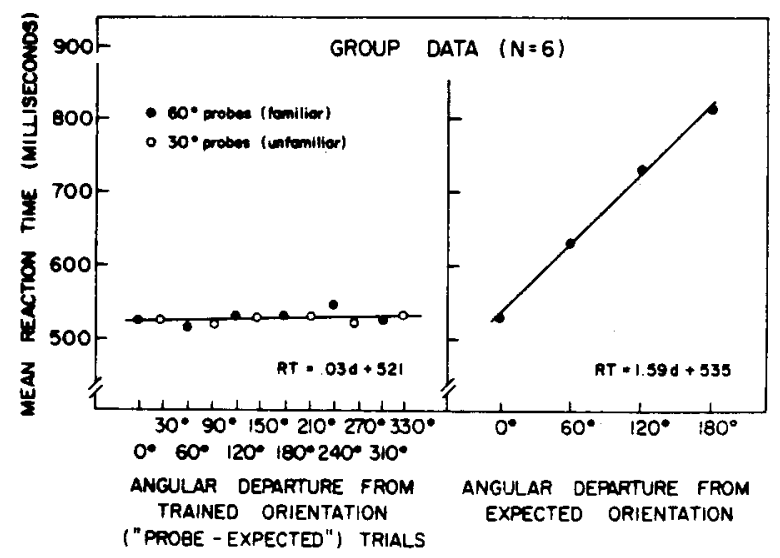

Figure 1. Reaction-time results for the group data. In the left-hand panel, mean reaction time is plotted as a function of angular departure of the test probe from the trained orientation for "probe-expected" triaks only. Open circles represent unfamiliar test probes, and solid circles represent familiar test probes. In the right-hand panel, mean reaction time is plotted as a function of angular departure of the test probe from the expected orientation for "probe-unexpected" trials.
Both standard and reflected versions were presented as test probes for $180^{\circ}$ departures from the expected orientation. For $60^{\circ}$ and $120^{\circ}$ departures, the trial composition was more complex. Since $60^{\circ}$ and $120^{\circ}$ departures could be in either a clockwise or a counterclockwise direction, one direction of angular departure was selected for presentation of the standard test form, and the other direction of angular departure was selected for presentation of the reflected test form. Assignment of test-form version to direction of angular departure was done as follows for each of the six subjects: For two of the test forms. standard test probes were presented at $60^{\circ}$ clockwise departures and at $120^{\circ}$ counterclockwise departures. Reflected test probes were presented at $60^{\circ}$ counterclockwise departures and at $120^{\circ}$ clockwise departures. For the third test form. this assignment of standard-reflected version to tirection of angular departure was reversed. The above assignment resulted in an equal number of standard and reflected test probes at each angular departure from the expected orientation, and it also permitted equal presentation of each of the three test forms in both standard and reflected versions at each of the six familiar orientations.

For each subject, the 252 experimental trials were presented in a random order and required approximately three $1-h$ sessions. Trials on which errors were made were retaken, interspersed with additional tiller trials.

\section{RESULTS}

\section{Reaction-Time Results}

The principal reaction-time results for the group data are illustrated in Figure 1. In the left-hand panel, mean reaction time-averaged over subjects, test forms, and standard-reflected versions-is plotted as a function of angular departure of the test form from the trained orientation for "probeexpected" trials only.

Note, in particular, two features of these data: (a) On "probe-expected" trials, average time required to discriminate standard from reflected test forms is virtualiy constant, regardless of the angular departure of the test form from a previously learned orientation. (b) Mean reaction times to test probes presented in unfamiliar orientations ( $30^{\circ}$ probes) are approximately equal to mean reaction times to probes presented in familiar orientations $\left(60^{\circ}\right.$ probes $)$. The range of mean reaction times plotted in the left-hand panel of Figure 1 is only $32 \mathrm{msec}$, and mean raction time to unfamiliar probes is not significantly different from mean reaction time to familiar probes $\left(t_{10}=.74\right.$, $\mathrm{p}>.20) .{ }^{1}$ In addition, though not illustrated in Figure 1. "standard" responses were faster than "reflected" responses at all test-form orientations. This standard-reflected difference averaged about $65 \mathrm{msec}$ for unfamiliar probes and $60 \mathrm{msec}$ for familiar probes.

In the right-hand panel of Figure 1, mean reaction time is plotted as a function of angular departure of the test form from the expected orientation. The $0^{\circ}$ point represents an average of all "probe-expected" trials (i.e.. all of the data points plotted in the left-hand panel of Figure 1). The $60^{\circ}, 120^{\circ}$, and $180^{\circ}$ points are averaged over subjects, test forms, angular departure from the trained orientation. standardreflected versions. and clockwise and counterclockuise angular departures. 
In marked contrast to the flat reaction-time function for "probe-expected" trials (ct. Figure 1. lefi-hand panel), mean reaction time to unexpected probes increases quite linearly with the angular departure of the test form from the expected orientation. A polynomial regression computed on this reaction-time function revealed a highly signiticant linear component $\mid F(1,2)=1963.69$, $\mathrm{p}<.001 \mid$ and no significant quadratic or higher order eflects: The slope of this reaction-time function. shown in the right-hand panel of Figure 1, compares well with the slope of the reaction-time function from the data of Cooper (1975), computed for just these six subjects and the appropriate test shapes. The values of the slopes from the two experiments are 1.59 and 1.74, respectively. Slopes for four of the subjects were smaller in the present experiment than in the earlier Cooper (1975) experiment, and slopes for two of the subjects were larger in the present experiment. A t test for a difference between the group slope in the present experiment and the group slope in the Cooper (1975) experiment proved nonsigniticant $\left(t_{5}=1.73\right.$, $p>.05)$. Finally, though not shown in the right-hand panel of Figure 1, "standard" responses were an average 52 msec faster than "reflected" responses on "probe-unexpected" trials.

Analyses of variance contirm the results for the group datal shown in Figure 1. Separate analyses of variance were pertormed on data for each of the trial types, viz, "probe-expected, familiar" trials, "probe-expected, unfamiliar" trials, and "probeunexpected" trials. Factors in the analyses of both types of "probe-expected" trials were "subjects," "angular departure from the trained orientation," and "standard-reflected versions." 2 For "probe- expected, unfamiliar" trials, only the main effects of "subjects" and "standard-retlected versions" were significant $\mid \mathrm{F}(5.25)=39.06, \mathrm{p}<.001$, and $\mathrm{F}(1,5)=$ 0. $40, p<.05$, respectively]. For "probe-expected. familiar" trials, these two main effects were again significant $\mid F(5,144)=151.43, \mathrm{p}<.001$, and $F(1,5)$ $=1472, p<.05]$, and, in addition, two interactions with the factor "subjects" were signiticant (for the Subject by Angular Departure from Trained Orientation interaction. $\mathrm{F}(25,144)=2.51, \mathrm{p}<.01$. and for the three-way interaction of these twe factors with the factor of "versions," $F(25,144) \neq 2.13$, $\mathrm{p}<.01)$.

Factors in the analysis of "probe-unexpected" trials were "subjects." "angular departure from the trained orientation," "angular depa:lure from the expected orientation," and "standard-retlected versions." The only two main effects to achieve statistical signiticance were "subjects" $[\mathrm{F}(5.50)=172.70, \mathrm{p}<.001]$ and "angular departure from the expected orientation" $[F(2,10)=30.50, p<.01]$. Note, in particular, that the main effect of "angular departure from the trained orientation" was not significant $[F(5,25)=$ 2.04, $p>.101$. In addition, the two-way interactions of Subjects by Angular Departure from the Trained Orientation and Subjects by Angular Departure from the Expected Orientation were significant $[\mathrm{F}(25,50)$ $=2.49, \mathrm{p}<.01$, and $\mathrm{F}(10,50)=2.36, \mathrm{p}<.05$, respectivelyl.

The data for each of the six individual subjects are illustrated in Figures 2 and 3. In Figure 2, mean reaction times for "probs-expected" trials are plotted as a function of angular departure of the test form from the trained orientation, corresponding to the group data shown in the left-hand panel of Figure 1.
Figure 2. Mean reaction time to unfamiliar and familiar expected probes, plotted as a function of angular departure from the trained orientation, for each of the six individual subjects. The functions for the individual subjects correspond to the group function illustrated in the left-hand panel of Figure 1.

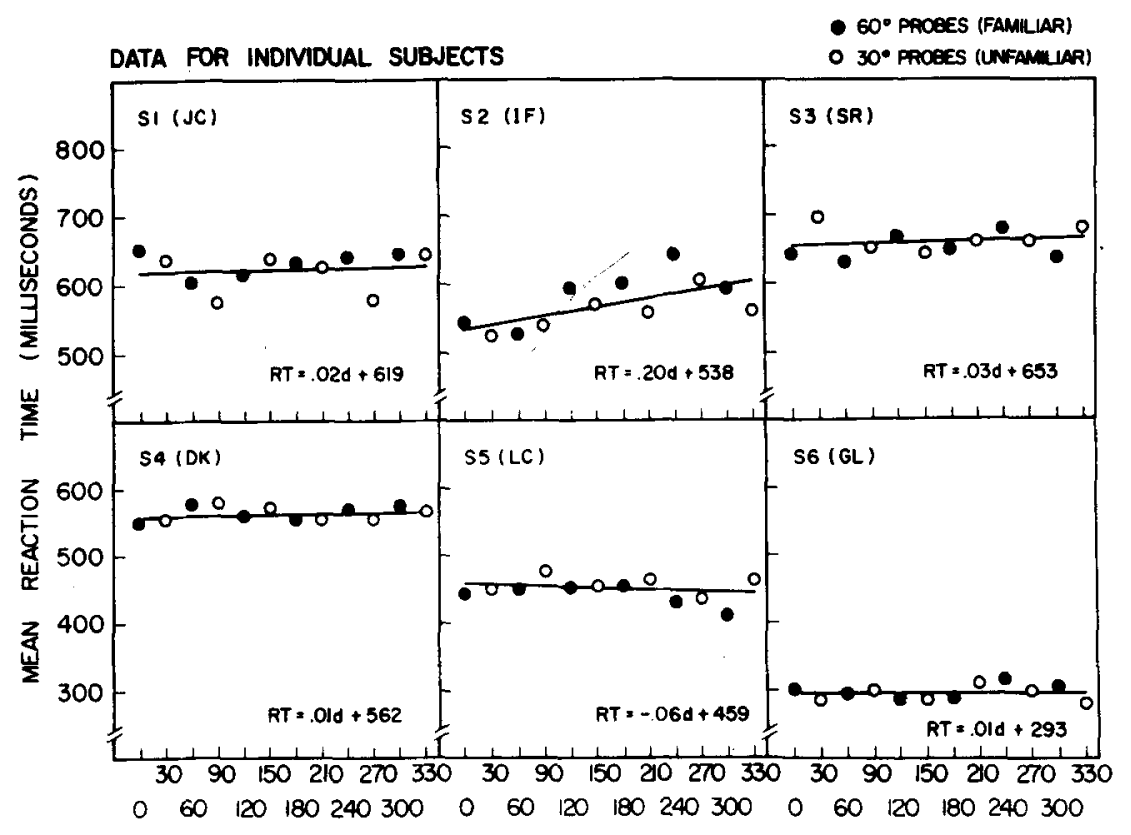

ANGULAR DEFARTURE OF PROBE FROM TRAINED ORIENTATION (DEGREES, CLOCKWISE) 


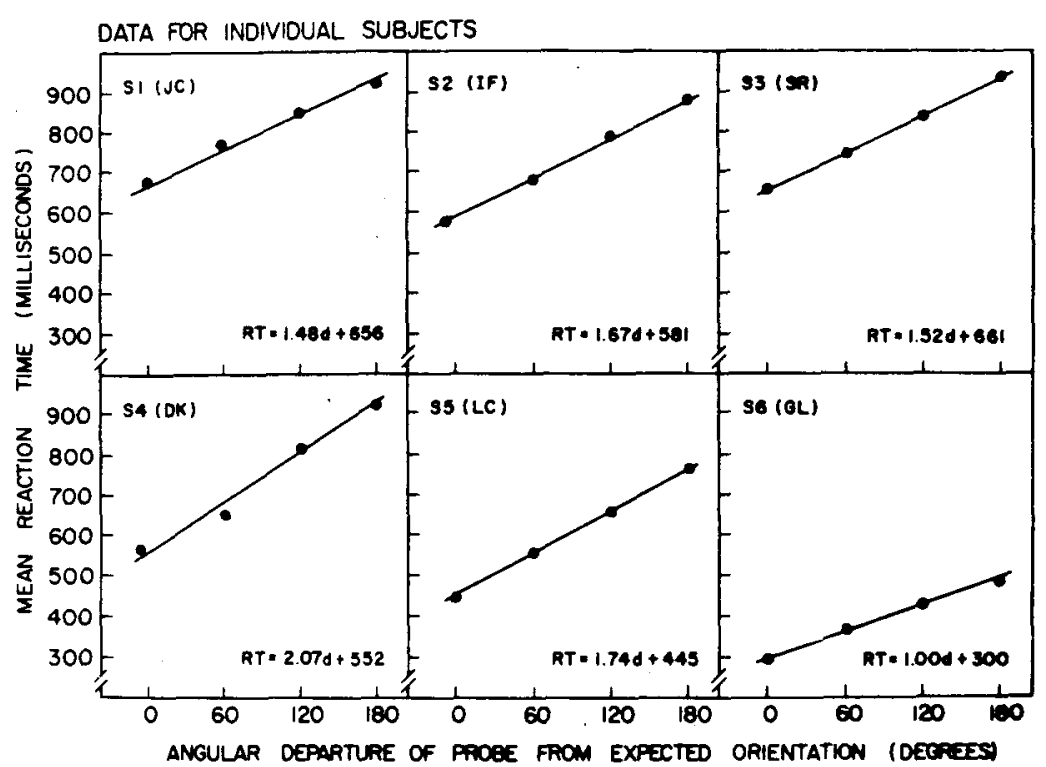

Figure 3. Mean reaction time to unexpected test probes, plotted as a function of angular departure of the test probe from the expected orientation, for each of the six individual subjects. The functions for the individual subjects correspond to the group function illustrated in the right-hand panel of Figure 1.
The central features of the group data are reflected in the data of each of the individual subjects. For all six subjects, reaction time to expected test probes is nearly constant for all test-form orientations. Best-fitting straight lines for each of the six reaction-time plots in Figure 2 show an appreciable positive slope for only one subject (Subject 2). In addition, mean reaction times to test probes presented in unfaniliar orientations are virtually indistinguishable from mean reaction times to test probes presented in familiar orientations. Mean reaction times to tamiliar and unfamiliar test probes were significantly different for only one of the individual subjects (for Subject 5, $t_{5}=2.30, p<.025$ ).

In Figure 3, mean reaction times for "probeunexpected" trials are plotted as a function of angular departure of the test probe from the expected orientation, corresponding to the group data shown in the right-hand panel of Figure 1. Again, the data for each of the individual subjects capture the essential features of the group data. For all six subjects, mean reaction time increases in a strikingly linear fashion with angular departure of the test form from the trained orientation. Though not illustrated in Figures 2 and 3, "standard" responses were faster than "reflected" responses for each of the six subjects and for both "probe-expected" and "probe-unexpected" trials.

\section{Errors}

Error rates for individual subjects ranged from $1.6 \%$ to $7.4 \%$, with a group average of $4.9 \%$. Average error rates for "unexpected, familiar" and "unexpected, unfamiliar" probes were approximately equal $-3.7 \%$ and $4.4 \%$, respectively. Average error rate for "unexpected" probes was $6.2 \%$. For both expected and unexpected probes, errors were distributed approximately equally over angular departures from the trained orientation. For unexpected probes, error rates increased monotonically with angular departure from the expected orientation.

\section{DISCUSSION}

The first significant feature of the data from the present experiment is the virtually constant reaction time to test probes presented in the expected orientation, regardless of the angular departure of that orientation from the previously trained position (cf. Figure 1, left-hand panel, for the group data and Figure 2 for the data of individual, subjects). These flat reaction-time functions can be compared with the striking increase in reaction time with angular departure from the trained orientation obtained by Cooper (1975) for these same subjects and same test forms. The difference between these two situations is that the procedure used in the present experiment permitted presentation of the test probe at an orientation for which the subject was maximally prepared and, hence, yielded essentially constant reaction times to probes at all angular departures from the trained orientation. Thus, the flat reaction-time function indicates that, during the mental rotation, the internal process passed through an ordered series of states which consisted of a sequence of "readinesses" to respond discriminatively to a particular stimulus presented in a particular external orientation.

The second and perhaps most significant aspect of the data presented in Figures 1 and 2 is that reaction times to familiar $\left(60^{\circ}\right.$ step) expected probes were no faster than reaction times to unfamiliar $\left(30^{\circ} \mathrm{step}\right)$ expected probes. This result provides evidence that 
the internal process which the subjects were carrying out in accordance with instructions was specifically one of rotation. in that the process passed through a trajectory of states which had a one-to-one correspondence to the intermediate stages in the rotation of an external object. That is. the near equivalence of reaction times to familiar and unfamiliar expected test probes indicates that, in rotating from a familiar orientation. A. to another familiar orientation. $C$. the internal process passed through an intermediate state corresponding to an unfamiliar orientation, B, at which the subject was most prepared for the presentation of a visual stimulus which he had never before viewed in that particular external orientation. If subjects were using some process other than a mental rotation such as, for example, successively generating representations of the standard shape in the familiar $60^{\circ}$ positions only, then we should expect reaction times to familiar probes to be uniformly shorter than reaction times to unfamiliar probes presented in intermediate orientations. $^{3}$

In marked contrast to the flat reaction-time functions obtained for "probe-expected" trials, reaction times to unexpected test probes increased linearly and quite sharply with angular departure of the test probe from the expected orientation (cf. Figure 1, right-hand panel, for the group data and Figure 3 for the data of individual subjects). These linear reaction-time functions suggest that subjects used a process of mental rotation in determining the "standard-retlected" version of unexpected test probes. In this case, subjects could have undertaken a correctional "poststimulus" rotation (cf. Cooper. 1975) in order to achieve congruence between the orientation of the external. unexpected test probe and the expected orientation. The representation of the standard shape presented as the identity cue could have been rotated. in a clockwise or counterclockwise direction. into the orientation of the test probe. and the two representations could then have been compared for a match or mismatch in shape. Alternatively, a representation of the test probe could have been rotated into the expected orientation. Additional evidence that a correctional "poststimulus" rotation was used on "probe-unexpected" trials derives from the fact that the values of the slopes of the reaction-time functions in Figure 3 are quite close to the slope values estimated for each of these six subjects in the Cooper (1975) rotation experiments.

The linear increase in reaction time with angular departure from the expected orientation on "probe-unexpected" trials has a further implication. In the absence of these data, any interpretation of the constant reaction times obtained on "probeexpected" trials would be somewhat problematic. That is, one could argue that the experienced subjects in the present experiment had learned to identify rotationally invariant features of the test shapes which served to distinguish standard from retlected versions. This explanation could account for the equivalence of reaction time to expected probes presented at all angular departures from the trained orientation; however. it also predicts that reaction time to unexpected probes should be rapid and independent of orientation. Thus, the flat reaction-time function obtained for "probe-expected" trials, in conjunction with the linear increase in reaction time obtained for "probe-unexpected" trials, provides strong support for the claim that a process of mental rotation was used both in preparing for the presentation of a test probe and in determining the version of a test probe presented in an unexpected orientation.

In summary, the results of the present experiment provide a demonstration of the analog nature of mental rotation. In particular, these results show that. during a mental rotation, the internal process passes through a trajectory of intermediate states which have a one-to-one correspondence to the intermediate stages in an external rotation. Each intermediate state through which the internal process passes consists of a readiness to respond discriminatively to a particular external object in a particular external orientation, if such an object were actually to be presented (cf. Cooper \& Shepard. 1973; Shepard. 1975).

On the basis of the data from the present experiment, it is not possible to determine whether the process of mental rotation is strictly continuous in nature or proceeds in a series of small, discrete steps. Experimental discrimination between these alternatives seems, at present. unfeasible. The equivalence of reaction times to familiar and unfamiliar expected probes suggests that the internal process may be continuous and, at the very least, indicates that the size of any discrete. component steps must be rather small (a maximum of $30^{\circ}$ ). In addition, the above result indicates that a mental rotation of at least $60^{\circ}$ is analog, in the sense that the process has an intermediate state which has the required one-to-one relationship to an intermediate stage in the rotation of an external object.

\section{REFERENCES}

Cooper. L. A. Mental rotation of random two-dimensional shapes. Cognitive Psychology. 1975. 7. 20.43.

COOPER, L. A., \& SHEPard, R. N. Chronometric studies of the rotation of mental images. In W. G. Chase (Ed.). Visual information processing. New York: Academic Press. 1973.

Metzler, J. Cognitive analogues of the rotation of threedimensional objects. Unpublished doctoral dissertation. Stantord University, 1973.

Metzler, J., \& Shepard, R. N. Transformational studies of the internal representation of three-dimensional objects. In $\mathbf{R}$. Solso (Ed.). Theorie's of cognitive psychology: The Lovola symposium. Potomac. Md: Lawrence Erlbaum. 1974. 
ShePard, R. N. Form, formation and transformation of internal representations. In $R$. Solso (Ed.), Information processing and cognition: The Loyola symposium. Potomac, Md: Lawrence Erlbaum, 1975.

Shepard, R. N., \& Metzler, J. Mental rotation of threedimensional objects. Science, 1971, 171, 701-703.

\section{NOTES}

1. The reported $t$ value is from a test which assumes that the two samples (mean reaction time to familiar and unfamiliar expected probes) are independent. An additional test for the difference between means of correlated samples was performed in which each mean reaction time to a familiar probe was paired with mean reaction time to the succeeding unfamiliar probe. Thus, the pairs of mean reaction times were to expected probes presented at $0^{\circ}$ and $30^{\circ} .60^{\circ}$ and $90^{\circ} .120^{\circ}$ and $150^{\circ}, 180^{\circ}$ and $210^{\circ} .240^{\circ}$ and $270^{\circ}$, and $300^{\circ}$ and $330^{\circ}$. For this test, $\mathrm{t}_{5}=.52, \mathrm{p}>.30$.

2. Since each of the six subjects used a different set of standard shapes, reaction times were averaged over the three standard shapes for each subject for the analyses of variance on the group data. In all analyses, the factor of "subjects" was treated as a random effect.

3. It could be argued that the equivalence of reaction times to familiar and unfamiliar expected probes resulted from the fact that, during the course of the experiment, subjects became "familiar" with probes presented in "unfamiliar" orientations. While it is difficult to evaluate this objection, three features of the present experiment render it unlikely. First, unfamiliar probes were presented on a small proportion of the trials (only 36 of the 252 experimental trials). Second, order of presentation of trials of all three types ("probe-expected, familiar," "probe-expected, unfamiliar," and "probe-unexpected") was random. Third, on each of the 36 "probe-expected, unfamiliar" trials, a unique combination of standard shape, test-form version, and probed orientation was presented.

(Received for publication October 6, 1975; revision received December 26, 1975.) 